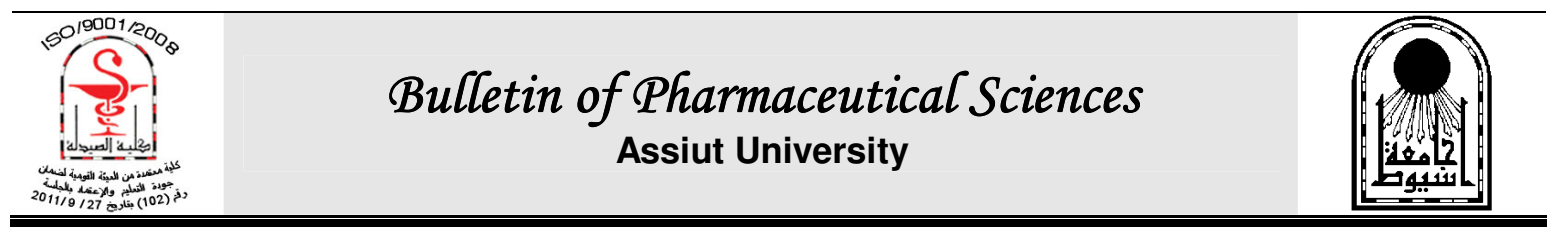

\title{
INTERACTIONS OF CELECOXIB WITH DIFFERENT ANTIDIABETIC DRUGS IN DIABETIC RATS
}

\author{
Manar A. Nader ${ }^{1,2 *}$, Shehta A. Said ${ }^{2}$ and Manar G. Helal ${ }^{2}$ \\ ${ }^{1}$ Department of Pharmacology and Toxicology, College of Pharmacy, Taibah University, El- \\ Madinah El-Munawarah, Saudi Arabia \& Faculty of Pharmacy, Mansoura University, \\ Mansoura, Egypt \\ ${ }^{2}$ Department of Pharmacology and Toxicology, Faculty of Pharmacy, Mansoura University, \\ Mansoura, Egypt
}

\begin{abstract}
The selective cyclooxygenase-2 inhibitor, celecoxib, can be prescribed to type 2 diabetic patients receiving oral hypoglycemic agents. This study was conducted to assess whether celecoxib affect the hypoglycemic activity of oral hypoglycemic agents in streptozotocininduced diabetic rats by measuring blood glucose level followed by determination of glucose uptake of isolated rat epididymal fat pads. Also, the effect of anti-diabetic agents on the antiinflammatory activity of celecoxib was also assessed using carrageenan-induced hind paw edema model in diabetic rats.This study revealed that the effect of celecoxib on the hypoglycemic activity of glibenclamide was additive and can be explained by stimulation of tissue glucose uptake by celecoxib. On the other hand, the effect of celecoxib on the hypoglycemic activity of glimepiride was not simple additive but synergistic. This synergistic effect can be explained not only by stimulation of tissue glucose uptake by celecoxib but also via a pharmacokinetic interaction by increasing the serum glimepiride concentration.
\end{abstract}

\section{INTRODUCTION}

With the availability of many over-thecounter non-steroidal anti-inflammatory drugs (NSAIDs) and several newer, selective cyclooxygenase-2 inhibitors (Coxibs), there are many choices for controlling acute and chronic pain and inflammation. Because many patients may administered NSAIDs in the presence of concurrent diseases and along with other drugs, the potential for drug interactions and enhanced organ toxicity should always be considered ${ }^{1}$. Coxibs were developed as a new class of NSAIDs. They were approved for the relief of acute pain and symptoms of chronic inflammatory conditions such as osteoarthritis (OA) and rheumatoid arthritis (RA). They have similar pharmacological properties but a slightly improved gastrointestinal safety profile if compared to traditional NSAIDs (Shi and Klotz, 2008). Celecoxib was the first of Coxibs class. It was approved in the United States in 1998 for relief of the signs and symptoms of $\mathrm{OA}$ and RA in adults ${ }^{2}$.

Since celecoxib is extensively metabolized by hepatic CYP2C9, plasma concentrations of the drug may be increased or decreased by CYP2C9 modifiers. Studies with celecoxib have identified a clinically significant drug interaction with the CYP2C9 inhibitor fluconazole ( $A U C$ increased by about 134\%) but not ketoconazole $^{3}$, phenytoin ${ }^{4}$ and methotrexate ${ }^{5}$. It also inhibits CYP2D6 and may affect plasma concentrations of CYP2D6 substrates, including $\beta$-adrenoceptor antagonists $^{6}$, antidepressants and antipsychotics ${ }^{7}$. It has long been known that high doses of salicylates lower plasma glucose concentrations $^{8}$. In addition, some NSAIDs, for example, ibuprofen can incidentally induce hypoglycaemia in diabetic patients who receive sulphonylurea therapy ${ }^{9}$. Celecoxib (200 mg, 
daily) increased insulin sensitivity in healthy subjects ${ }^{10}$. These observations imply that Coxibs may affect the regulation of glucose homeostasis in normal and insulin resistant subjects. However, the underlying mechanism(s) through which NSAIDs affect plasma level of glucose remain unclear.

In the present study, two diseases (type 2 diabetes mellitus and inflammatory disease) that may co-exist and require chronic treatment were considered and the occurrence of interaction between the concurrently used drugs was assessed. In this study, two sulphonylureas were selected, glibenclamide and glimepiride. They are different in their duration of action and metabolic pathway ${ }^{11}$. Sitagliptin was also included in this interaction study with celecoxib as it is a newer oral antidiabetic agent and few data about its interactions are available.

\section{MATERIAL AND METHODS}

\section{Animals}

Adult Sprague-Dawley rats weighing 150$200 \mathrm{~g}$ were purchased from "Egyptian Organization for Biological Products and Vaccines", Giza, Egypt. Animals were maintained under standard conditions of temperature about $25 \pm$ $2^{\circ} \mathrm{C}$ with $12 \mathrm{hrs}$ light/dark cycle and kept at constant environmental and nutritional conditions throughout the experimental period. The rats were allowed free access to standard laboratory food and water, ad libitum. All animals were acclimatized to the laboratory environment for 2 weeks and were fasted 12 hrs prior to experimentation. The animal experiments described below comply with the ethical principles and guidelines for the care and use of laboratory animals adopted by the "Ethical Research Committee" of Faculty of Pharmacy, Mansoura University.

\section{Drugs and Chemicals}

Celecoxib gifted from Amoun Pharmaceutical Industrial Co., El-Obour City, Egypt. Glibenclamide gifted from PHARCO Pharmaceuticals, Alexandria, Egypt. Glimepiride was gifted from Sedico Pharmaceutical Co., 6 th October City, Egypt. Sitagliptin obtained from Merck \& Co. Inc., New Jersy, USA.Regular human insulin (Humulin vial, $100 \mathrm{U} / \mathrm{ml}$ human insulin), Lilly Egypt under the license of Eli Lilly USA,
Indiana, USA.STZ, carrageenan, bovine serum albumin fraction $\mathrm{V}$, acetonitrile (HPLC grade), water (HPLC grade), methanol (HPLC grade) and dichloromethane purchased from Sigma Chemical Co., St. Louis, MO., USA. All the other used chemicals are of fine analytical grade.

\section{Experimental protocol Induction of diabetes}

Mild to moderate diabetes induced by single intraperitoneal (IP) injection of STZ at a dose of $40 \mathrm{mg} / \mathrm{kg}$ (dissolved in citrate buffer, $\mathrm{pH}=4.5)$ after 12 hour fasting. STZ-injected animals were allowed to drink $10 \%$ glucose solution for 12-24 hrs after STZ injection to prevent initial STZ-induced hypoglycemic mortality. Ninety six hours after injection of STZ, hyperglycemia was confirmed by determination of fasting serum glucose level. One week after injection of STZ, rats with fasting serum glucose between 180-260 mg/dl were considered mild to moderate diabetic and used in further experiments.

After confirmation of diabetes using glucose oxidase kit purchased from Fortress Diagnostics Limited, Belfast, UK., diabetic rats were randomly divided into 8 groups of 6 rats each. Group (1): received the vehicle $(2 \mathrm{ml} / \mathrm{kg}$ $0.5 \%$ CMC, orally). Group (2): received celecoxib (40 mg/kg, orally) ${ }^{12}$. Group (3): received glibenclamide $(3 \mathrm{mg} / \mathrm{kg} \text {, orally })^{13}$. Group (4): received glimepiride (2 $\mathrm{mg} / \mathrm{kg}$, orally) ${ }^{14}$.Group (5): Sitagliptin-treated group, rats received Sitagliptinin a dose of $3 \mathrm{mg} / \mathrm{kg}^{15}$. Group (6): received celecoxib (40 mg/ $\mathrm{kg}$, orally as a single dose) $1 \mathrm{hr}$ after Glibenclamideadministration ( $3 \mathrm{mg} / \mathrm{kg}$, orally). Group (7): received celecoxib (40 mg/kg, orally as a single dose) $1 \mathrm{hr}$ after Glimepiride administration (2 mg/kg, orally). Group (8): celecoxib + sitagliptin-treated group, rats received celecoxib (40 $\mathrm{mg} / \mathrm{kg})$ and sitagliptin(3 $\mathrm{mg} / \mathrm{kg}$ ). This dose regimen and time of blood sampling were selected as the optimum and proper conditions for this study after previous performed trial experiments (data not included). The main finding of these experiments is that maximum interaction between the tested drugs could be observed when celecoxib was given $1 \mathrm{hr}$ after antidiabetic administration in these selected doses. Two hours after administration of antidiabetic agents, rats were injected subcutaneously with 
$0.05 \mathrm{ml}$ of $1 \%$ carrageenan into the plantar tissue of the right hind paw. The left hind paw of each rat received a subplantar injection of equal volume of normal saline ${ }^{16}$. Blood samples were collected 2, 3, 4, 5 and $6 \mathrm{hrs}$ following the administration of each antidiabetic drug for determination of serum glucose according to the method described by Trinder ${ }^{17}$. Twenty four hours after carrageenan injection; rats were killed then the right and the left hind paws of each animal were cut and weighed for calculation of percentage inhibition in paw edema according to the method previous ${ }^{16}$.

\section{Effect of celecoxib on glucose uptake using isolated rat epididymal fat pads}

Epididymal fat pads from fasted nondiabetic or diabetic male rats weighing about $200 \mathrm{~g}$ were used. The rats were anaesthetized with ether and the epididymal fat pads were isolated according to the method described by Rodbell $^{18}$ and incubated with Kerb's Ringer phosphate buffered solution containing glucose $(2 \%)$ and bovine albumin $(3 \%)^{19}$. Kerb's Ringer phosphate buffered solution was freshly prepared and aerated with $95 \%$ O2 containing $5 \% \mathrm{CO} 2$ for 5 minutes and the $\mathrm{pH}$ of the medium was adjusted at 7.4 by $1 \mathrm{~N}$ sodium hydroxide solution. Celecoxib was prepared as $0.05 \%$ solution in $95 \%$ ethanol. This solution was diluted by Kerb's Ringer phosphate buffer to obtain a concentration of $1.8 \mu \mathrm{M}$. The pieces of adipose tissue isolated from non-diabetic or diabetic rats were divided into 8 groups (each composed of 6 tubes) and incubated with control solution $(2 \mathrm{ml}$ ethanol\% in the incubation medium), celecoxib $(1.8 \mu \mathrm{M})$, insulin $\left(10^{-7} \mathrm{M}\right)^{20}$ or celecoxib $(1.8 \mu \mathrm{M})$ +insulin $\left(10^{-7} \mathrm{M}\right)$ combination. The isolated tissue was placed in specific screw-capped tubes which were incubated in metabolic shaker at $37^{\circ} \mathrm{C}$ for $1 \mathrm{hr}$ with a shaking rate of $100 \mathrm{rpm}$. Then the pads were taken out and aliquots were taken from the medium and used for the assay of glucose concentration in the medium to determine the amount of glucose utilized by the used adipose tissue.

\section{Effect of celecoxib on the pharmacokinetics of glimepiride}

Serum glimepiride level was determined using HPLC technique according to the method described by Rabbaa-Khabbaz et al. ${ }^{21}$. Freshly prepared suspensions of glimepiride and celecoxib were administered as single oral dose to 2 groups of male overnight-fasted STZinduced diabetic rats $(n=30)$. Group I received glimepiride $(2 \mathrm{mg} / \mathrm{kg}$, orally), group II received celecoxib (40 $\mathrm{mg} / \mathrm{kg}$, orally) $1 \mathrm{hr}$ after glimepiride administration $(2 \mathrm{mg} / \mathrm{kg}$, orally). Then each group was subdivided into 6 subgroups (each composed of 6 rats) where each subgroup was used for blood sampling once at only 1 time interval. Blood samples (5 $\mathrm{ml}$ each) from each subgroup were collected from retro-orbital plexus, at $0 \mathrm{~min}$ (pre-dose), 1, 2, 3, 4 and 5 hrspostoralglimepiride dosing. Blood samples were centrifuged at 1000 gfor 15 min to obtain the serum. Separation was performed with a ShimadzuTM LC-20A Series Chromatograph equipped with a Rheodyne injector valve with a $20 \mu 1$ loop - 10 - and a SPD-20A UV detector operated at $228 \mathrm{~nm}$. LC workstation (Nishinokyo-Kuwabaracho, Nakagyoku, Kyoto 604-8511, Japan) for data collection and processing.Separation was achieved on a Shim-pack VP-ODS column (5 $\mu \mathrm{m})(150 \mathrm{X} 4.6 \mathrm{~mm})$ combined with a guard column (Nishinokyo-Kuwabaracho, Nakagyoku, Kyoto 604-8511, Japan). The mobile phase consisted of $40 \%$ water acidified with glacial acetic acid $(0.1 \mathrm{mM}, \mathrm{pH}=2.6)$ and $60 \%$ acetonitrile. The flow rate was 0.7 $\mathrm{mL} / \mathrm{min}$. Glibenclamidewas used as internal standard and prepared by dissolving $15 \mathrm{mg}$ in $25 \mathrm{ml}$ methanol. Then this solution was further diluted $1 / 10$ with methanol. The retention times for glimepiride and glibenclamidewere $7 \& 6$ min, respectively. An internal standard solution of $50 \mathrm{~mL}$ was added to $1.5 \mathrm{~mL}$ serum and mixed for 10 seconds on vortex. $\mathrm{HCl} 1 \mathrm{~N}$ (40 $\mu \mathrm{L})$ was immediately added while gently vortexing the tubes. To each tube, dichloromethane $(7 \mathrm{ml})$ was added and the tubes were vortexed for $10 \mathrm{sec}$. The sample was finally shaken on a rotating shaker (30 rotation/ minute) for $30 \mathrm{~min}$. After centrifugation for $20 \mathrm{~min}$ at $1000 \mathrm{~g}$ and $4^{\circ} \mathrm{C}, 6$ $\mathrm{ml}$ of the clear dichloromethane layer was transferred to a glass tube and evaporated under nitrogen. The dried residue was taken up with $0.5 \mathrm{~mL}$ of mobile phase and $20 \mu \mathrm{L}$ of this mixture were injected.Data were generated by linear regression of the terminal phase of serum concentration-time curve for log concentration 
against time. Pharma-cokinetic parameters of glimepiride either alone or in combination with celecoxib were characterized by peak concentration in serum $\left(C_{\max }\right)$, concentration peak time $\left(t_{\max }\right)$, area under the concentrationtime curve - 11 - $(A U C)$, elimination rate constant $(\mathrm{Ke})$, clearance $(C L)$, and volume of distribution $\left(V_{d}\right)$.

\section{Statistical analysis}

Data are expressed as mean \pm S.E.M. Comparison between two different groups was carried out using Student's t test. Comparison between more than two different groups was carried out using one way ANOVA followed by Tukey-Kramer multiple comparisons test. Paired Student's t-test was used as a test of significance for comparison between two arithmetic means of the same group before and after treatment.Statistical tests were carried out using GraphPad Instat computer program V 3.10 (GraphPad Software Inc, San Diego, CA, USA). Significance was set at $\mathrm{p}<0.05$.

\section{RESULTS AND DISCUSSION}

\section{Results}

\section{Effect on serum glucose level}

Oral administration of $0.5 \% \mathrm{CMC}$ showed significant increase in serum glucose level of diabetic rats after $2,3,4,5$ and $6 \mathrm{hrs}$ of administration when compared to their corresponding mean initial value.Treatment of diabetic rats with celecoxib showed significant decrease in serum glucose level (12.4\%) $1 \mathrm{hr}$ after celecoxib treatment when compared to the corresponding mean initial value and significantly decreased serum glucose level after $1,2 \& 3 \mathrm{hrs}$ when compared to the corresponding mean values of the control group. Diabetic rats treated with glibenclamide demonstrated significant decrease in serum glucose level after 2, 3, 4, 5 and $6 \mathrm{hrs}$ of treatment when compared to both their corresponding mean initial value and the corresponding mean values of the control group (Fig. 1A). Glimepiride treatment showed significant decrease in serum glucose level in diabetic rats after 2, 3 and $4 \mathrm{hrs}$ of treatment when compared to their corresponding mean initial value and it significantly decreased serum glucose level after 2, 3, 4, 5 and $6 \mathrm{hrs}$ when compared to the corresponding mean values of the control group (Fig. 1B). Treatment of diabetic rats with sitagliptin significantly decreased serum glucose level after 2, 3, 4, 5 and $6 \mathrm{hrs}$ of treatment when compared to both their corresponding mean initial value and the corresponding mean values of the control group (Fig. 1C).

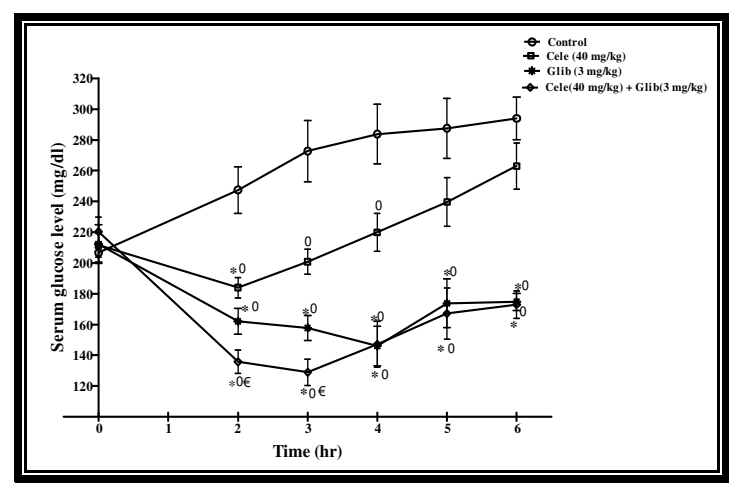

Fig. 1A: Effect of celecoxib (Cele), glibenclamide (Glib) and their combination on serum glucose level in STZ-induced diabetic rats.

Glib (3 $\mathrm{mg} / \mathrm{kg}$, orally) was given at time zero, Cele(40 mg/kg, orally) was given after $1 \mathrm{hr}$. Measurements of serum glucose level were done before and 2, 3, 4, $5 \& 6 \mathrm{hrs}$ after treatment with antidiabetic drug.

Values represent the mean \pm SEM of 6 animals.

*Significantly different from its initial value using paired student's t-test at $(\mathrm{p}<0.05)$.

$0, €$ Significantly different from the corresponding mean values of the control group or the Glib-treated group, respectively, using one way ANOVAfollowed by Tukey-Kramer multiple comparisons test $(\mathrm{p}<$ $0.05)$.

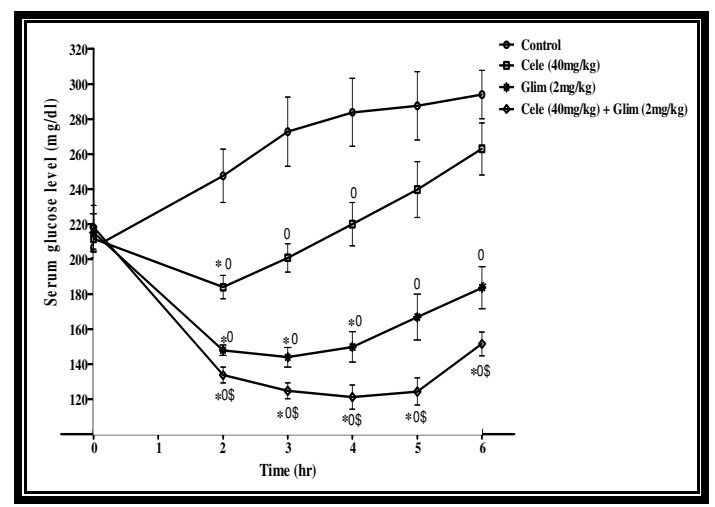

Fig. 1B: Effect of celecoxib (Cele), glimepiride (Glim) and their combination on serum glucose level in STZ-induced diabetic rats. 
Glim ( $2 \mathrm{mg} / \mathrm{kg}$, orally) was given at time zero, Cele (40 mg/kg, orally) was given after $1 \mathrm{hr}$. Measurements of serum glucose level were done before and 2, 3, 4, $5 \& 6$ hrs after treatment with antidiabetic drug.

Values represent the mean \pm SEM of 6 animals.

* Significantly different from its initial value using paired student's t-test at $(\mathrm{p}<0.05)$.

$0, \$$ Significantly different from the corresponding mean values of the control group or the Glim-treated group, respectively, using one way ANOVA followed by Tukey-Kramer multiple comparisons test $(\mathrm{p}<0.05)$.

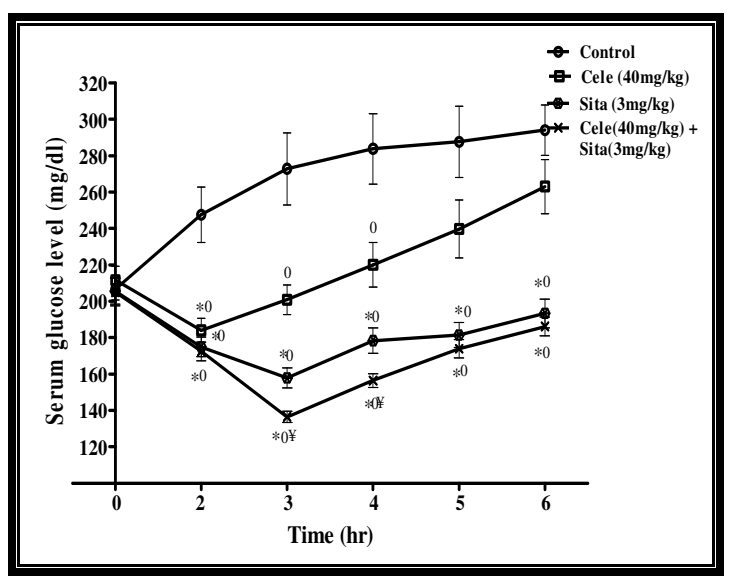

Fig. 1C: Effect of celecoxib (Cele), sitagliptin (Sita) and their combination on serum glucose level in STZ-induced diabetic rats. $\mathrm{Sita}(3 \mathrm{mg} / \mathrm{kg}$, orally) was given at time zero, Cele(40 mg/kg, orally) was given after $1 \mathrm{hr}$. Measurements of serum glucose level were done before and 2, 3, 4, 5 \& 6 hrs after treatment with antidiabetic drug. Values represent the mean \pm SEM of 6 animals.

* Significantly different from its initial value using paired student's t-test at $(\mathrm{p}<0.05)$.

0 , $¥$ Significantly different from the corresponding mean values of the control group or the Sita-treated group, respectively, using one way ANOVA followed by Tukey-Kramer multiple comparisons test $(\mathrm{p}<0.05)$.

In celecoxib + glibenclimide combination group, serum glucose level significantly decreased after 2, 3, 4, 5 and 6 hrs when compared to both the corresponding initial value and the corresponding mean values of the control group. This combination showed a significant decrease in serum glucose level after 2 and 3 hrs of treatment when compared to the corresponding mean values of glibenclamide-treated group. In diabetic group treated with celecoxib + glimepiride combination, serum glucose level significantly decreased after 2, 3, 4, 5 and 6 hrs of treatment when compared to the corresponding initial value, the corresponding mean values of the control group and the corresponding mean values of glimepiride -treated group. Diabetic rats treated with celecoxib + sitagliptin combination showed significant decrease in serum glucose level after 2, 3, 4, $5 \& 6 \mathrm{hrs}$ of treatment when compared to both the corresponding mean initial value and the corresponding mean values of the control group. This combination demonstrated a significant decrease in serum glucose level after 3 \& 4 hrs when compared to the corresponding mean values of sitagliptin treated group.

\section{Effect on carrageenan-induced hind paw edema}

Intraplantar injection of carrageenan in diabetic rats showed an inflammatory response that was characterized by an increase in right hind paw weight (indicative of paw edema) when compared to the weight of contralateral (saline-injected) hind paw.Pretreatment of carrageenan-injected diabetic rats with celecoxib significantly reduced the carrageenan-induced paw edema when compared to the mean value of the control group.Carrageenan-injected diabetic rats pretreated with glibenclamide, glimepiride or sitagliptin showed non-significant change in carrageenan-induced paw edema when compared to the mean value of the control group.Pretreatment of carrageenan-injected diabetic rats with combination of celecoxib + glibenclamide showed significant reduction in carrageenan-induced paw edema when compared to the mean value of the control group. However, there was no significant change in paw edema when compared to the mean value of celecoxib-treated group. Pretreatment of carrageenan-injected diabetic rats with celecoxib + glimepirideor celecoxib+ sitagliptin combinations showed significant reduction in paw edema when compared to the mean value of the control group with nonsignificant change in paw edema when 
compared to the mean value of celecoxib treated group (Fig. 2).

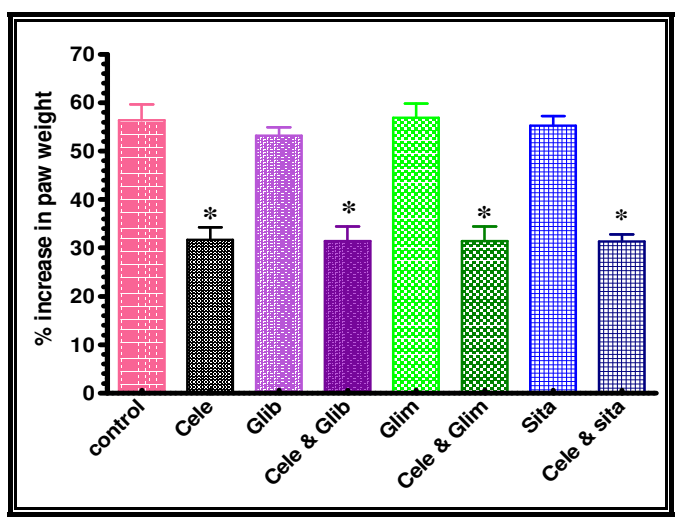

Fig. 2: Effect of celecoxib (Cele) either alone or in combination with glibenclamide (Glib), glimepiride (Glim) or sitagliptin (Sita) on carrageenan-induced hind paw edema in diabetic rats.

Glib (3 mg/kg, orally), Glim (2 mg/kg, orally) and Sita $(3 \mathrm{mg} / \mathrm{kg}$, orally) were given $2 \mathrm{hrs}$, while Cele $(40$ $\mathrm{mg} / \mathrm{kg}$, orally) was given $1 \mathrm{hr}$ before carrageenan injection. Measurement of paw weight was done 4 hrs after carrageenan injection.

Values represent the mean \pm SEM of 6 animals.

*Significantly different from the corresponding mean value of the control group using one way ANOVA followed by Tukey-Kramer multiple comparisons test $(\mathrm{p}<0.05)$.

Effect on glucose uptake using isolated nondiabetic and diabetic rat epididymal fat pads

The amounts of glucose utilized by isolated non-diabetic and diabetic rat epididymal fat pads incubated with control solution were 1.11 and $0.24 \mathrm{mg}$ glucose $/ \mathrm{g}$ wet tissue/hr, respectively. Addition of insulin $(0.1 \mu \mathrm{M})$ to the incubation media showed significant increase in the amount of glucose utilized by both isolated non-diabetic and diabetic rat epididymal fat pads when compared to the mean values of the corresponding control non-diabetic and diabetic groups, respectively. Incubation of epididymal fat pads with celecoxib $(1.8 \mu \mathrm{M})$ showed significant increase in the amounts of glucose uptake by both isolated non-diabetic and diabetic epididymal fat pads when compared to the mean values of the corresponding control non-diabetic and diabetic groups, respectively. Incubation of isolated non amount of glucose uptake when compared to the mean value of the corresponding insulin-treated group.The amount of glucose utilized by diabetic rat fat pads significantly increased when compared to the mean value of the corresponding insulin-treated group. Incubation of both isolated non-diabetic and diabetic rat epididymal fat pads with incubation medium containing celecoxib $(2.6 \mu \mathrm{M})$ demonstrated significant increase in the amount of glucose uptake when compared to the mean values of the corresponding control and insulin-treated groups. Isolated non-diabetic and diabetic rat epididymal fat pads incubated with insulin $(0.1 \mu \mathrm{M})+$ celecoxib $(1.8$ or $2.6 \mu \mathrm{M})$ demonstrated significant increase in glucose utilization in both non-diabetic and diabetic rats when compared to the mean values of their corresponding control, insulin-treated and celecoxib treated groups.

Table 1: Effect of celecoxib either alone or in combination with insulin on glucose uptake using isolated non-diabetic and diabetic rat epididymal fat pads.

\begin{tabular}{|l|c|c||}
\hline \multirow{2}{*}{} & \multicolumn{2}{|c|}{ Glucose uptake (mg glucose/ g wet tissue/ hr) } \\
\cline { 2 - 3 } & Non-diabetic group & Diabetic group \\
\hline Control & $1.11 \pm 0.08$ & $0.24 \pm 0.03$ \\
\hline Insulin $(0.1 \mu \mathrm{M})$ & $2.17 \pm 0.11 \$$ & $0.56 \pm 0.02 \mu$ \\
\hline Celecoxib $(1.8 \mu \mathrm{M})$ & $1.46 \pm 0.08 \$ @$ & $0.74 \pm 0.06 \mu ¥$ \\
\hline Celecoxib $(2.6 \mu \mathrm{M})$ & $2.78 \pm 0.15 \$ @$ & $1.23 \pm 0.07 \mu ¥$ \\
\hline $\begin{array}{l}\text { Celecoxib }(1.8 \mu \mathrm{M})+ \\
\text { Insulin }(0.1 \mu \mathrm{M})\end{array}$ & $4.41 \pm 0.25 \$ @ *$ & $2.08 \pm 0.13 \mu ¥ £$ \\
\hline $\begin{array}{l}\text { Celecoxib }(2.6 \mu \mathrm{M})+ \\
\text { Insulin }(0.1 \mu \mathrm{M})\end{array}$ & $5.26 \pm 0.12 \$$ @* & $2.96 \pm 0.18 \mu ¥ £$ \\
\hline
\end{tabular}

Values represent the mean \pm SEM of 6 isolated non-diabetic or diabetic rat epididymal fat pads.

$\$ \mu$ significantly different from the mean values of the non-diabetic and diabetic control groups, respectively, using unpaired student's t-test at $(\mathrm{p}<0.05)$.

@ $¥$ significantly different from the mean values of the non-diabetic and diabetic Ins-treated groups, respectively, using unpaired student’s t-test at $(\mathrm{p}<0.05)$. 
*, $£$ Significantly different from the mean values of the non-diabetic and diabetic corresponding Celecoxobtreated groups, respectively, using unpaired student's t-test at $(\mathrm{p}<0.05)$.

Effect on the pharmacokinetics of glimepiride

The mean values of serum concentration $(\mathrm{ng} / \mathrm{ml})$ for glimepiride alone after 1, 2, 3, 4 \& $5 \mathrm{hrs}$ were found to be $710,1330,1145,800 \&$ $597 \mathrm{ng} / \mathrm{ml}$, respectively. In case of glimepiride with celexocib co-administration, serum glimepiride concentrations were found to be $705,1461,1584,1150 \& 839 \mathrm{ng} / \mathrm{ml}$ after 1,2 , $3,4 \& 5$ hrs, respectively. Treatment with celecoxib moderately increased serum glimepiride concentrations. Co-administration of celecoxib with glimepiride showed 10, 38, $44 \& 40 \%$ increase in glimepiride concentrations after 2, 3, 4 \& 5 hrs, respectively. This combination showed significant increase in the mean values of serum glimepiride concentration after 2, 3, 4 $\& 5$ hrs. of treatment when compared to the corresponding mean values of glimepiride treated group. The mean value of the time to reach peak serum concentration $\left(t_{\max }\right)$ for glimepiride alone was found to be $2 \mathrm{hrs}$ and when glimepiride was co-administered with celexocib, the mean value of $t_{\max }$ was found to be $3 \mathrm{hrs}$. The mean value of peak serum glimepiride concentration $\left(C_{\max }\right)$ for glimepiride alone was found to be $1330 \mathrm{ng} / \mathrm{ml}$; co-administration of glimepiride with celecoxib resulted in a $C_{\max }$ of $1584 \mathrm{ng} / \mathrm{ml}$ which was almost a $19 \%$ increase when compared to the corresponding mean value of glimepiridetreated group. The mean value of area under the serum concentration-time curve $(A U C)$ for glimepiride alone was found to be $4303 \mathrm{ng} \mathrm{ml}^{-1}$ $\mathrm{hr}$, when glimepiride was co-administered with celexocib, $A U C$ was found to be $5361 \mathrm{ng} \mathrm{ml}^{-1}$ $\mathrm{hr}$, which was significantly different and showed about 1.2 fold increase when compared to glimepiride-treated group. The mean value of apparent oral glimepiride clearance $(C L / F)$ and apparent volume of distribution $\left(V_{d} / F\right)$ for glimepiride alone were found to be $465 \mathrm{ml} \mathrm{hr}^{-1}$ $\mathrm{kg}^{-1}$ and $1349 \mathrm{ml} \mathrm{kg}^{-1}$, respectively. When glimepiride was co-administered with celexocib, the $C L / F$ and $V_{d} / F$ were $373 \mathrm{ml} \mathrm{hr}^{-1}$ $\mathrm{kg}^{-1}$ and $1577 \mathrm{ml} \mathrm{kg}^{-1}$, respectively, which were significantly different when compared to glimepiride-treated group. The mean value of elimination rate $\operatorname{constant}\left(K_{e}\right)$ for glimepiridealone was found to be $0.35 \mathrm{hr}^{-1}$, glimepiride when co-administered with celecoxib showed a $K_{e}$ of $0.24 \mathrm{hr}^{-1}$ which was significantly different when compared to the corresponding mean value of glimepiridetreated group. The mean value of elimination half-life time $\left(t_{1 / 2}\right)$ for glimepiride alone was found to be $2.18 \mathrm{hrs}$, co-administration of glimepiride with celecoxib resulted in a $t_{1 / 2}$ of $2.7 \mathrm{hrs}$ which was significantly different when compared to the corresponding mean value of glimepiride-treated group.

Table 2: Effect of celecoxib on pharmacokinetic parameters of glimepiridein STZ-diabetic rats.

\begin{tabular}{|c|c|c|}
\hline \multicolumn{2}{|l|}{ Treatment } & \multirow{2}{*}{$\begin{array}{c}\text { Pharmacokinetic } \\
\text { parameters }\end{array}$} \\
\hline Glimepiride + Celecoxib & Glimepiride & \\
\hline $1584 \pm 37^{\epsilon}$ & $1330 \pm 29$ & $C_{\max }(\mathrm{ng} / \mathrm{ml})$ \\
\hline $0.24 \pm 0.02^{\epsilon}$ & $0.35 \pm 0.03$ & $K_{e}\left(\mathrm{hr}^{-1}\right)$ \\
\hline $2.7 \pm 0.09^{\epsilon}$ & $2.18 \pm 0.08$ & $t_{1 / 2}(\mathrm{hr})$ \\
\hline $5361 \pm 47^{\epsilon}$ & $4303 \pm 69$ & $A U C\left(\mathrm{ng} \mathrm{ml}^{-1} \mathrm{hr}\right)$ \\
\hline $373 \pm 3^{€}$ & $465 \pm 8$ & $C L / F\left(\mathrm{ml} \mathrm{hr}^{-1} \mathrm{~kg}^{-1}\right)$ \\
\hline $1577 \pm 12^{\epsilon}$ & $1349 \pm 22$ & $V_{d} / F\left(\mathrm{ml} \mathrm{kg}^{-1}\right)$ \\
\hline
\end{tabular}

$C_{\max }$ is the peak serum concentration, $K e$ is the elimination rate constant, $t_{1 / 2}$ is the elimination half life time, $A U C$ is the area under the concentration-time curve, $C L / F$ is the apparent oral clearance, $V_{d} / F$ is the apparent volume of distribution.

Values represent the mean \pm SEM of 6 rats.

$(€)$ Significantly different from the corresponding mean value of the glimepiride treated group using unpaired Student's t-test at $(\mathrm{p}<0.05)$. 


\section{Discussion}

The oral administration of the vehicle ( $0.5 \%$ CMC) containing no hypoglycaemic agent to diabetic rats provoked a significant rise in serum glucose concentration after 2,3 , $4,5 \& 6 \mathrm{hrs}$. CMC-induced increase in serum glucose concentration observed in this study coincides with the previous report of Ladriere et $a l^{22}$.These results may be explained on the basis of CMC is polysaccharide and is digested in-vivo to give glucose or the release of inflammatory mediators following carrageenan injection increase blood glucose level ${ }^{23}$.

Treatment of diabetic rats with celecoxib significantly decreased the serum glucose level after $2,3 \& 4$ hrs compared to the control group. Administration of glibenclamide produced maximum antidaiabetic activity after $4 \mathrm{hrs}$ of administration while glimepiride and sitagliptin produced maximum decrease in serum glucose level at $3 \mathrm{hrs}$. after its administration. Co-administration of glibenclamide and celecoxibto diabetic rats increased the hypoglycaemic activity and accelerated the hypoglycaemic activity peak of glibenclamide which occurred at 3 hrs. Treatment of diabetic rats with celecoxiblhr. after administration of sitagliptin resulted in significant decrease in serum glucose after $3 \&$ $4 \mathrm{hrs}$. of sitagliptin administration when compared to sitagliptin-treated group. Administration of glimepirideto diabetic rats followed by celecoxib after $1 \mathrm{hr}$ produced significant increase in glucose lowering activity of glimepiride which was persisted for $6 \mathrm{hrs}$ after glimepiride administration when compared to glimepiride treated group. Treatment of STZ-induced diabetic rats with single oral dose of celecoxib produced significant decrease in fasting serum glucose level (by about 12\%). Significant hypoglycemic activity of celecoxib was maintained for $3 \mathrm{hrs}$ with peak activity at $1 \mathrm{hr}$ after its administration to diabetic rats. This result is in agreement with Gonzalez-Ortiz et $a l .{ }^{10}$; Giugliano et $a l .{ }^{24}$.

Previous studies have shown that COX derivatives, mainly prostaglandin $\mathrm{E}_{2}\left(\mathrm{PGE}_{2}\right)$, reduce the insulin response to glucose and NSAIDs enhance the insulin response to various stimuli ${ }^{25} . \mathrm{PGE}_{2}$ also decreases the expression of glucose transport protein-4 (GLUT4) and decreases the tissue glucose uptake and insulin sensitivity as well as the inhibition of COX-2 and subsequent prevention of prostaglandin formation by NSAIDs improves tissue insulin sensitivity ${ }^{26}$.

Accordingly, it was hypothesized that, the hypoglycemic activity of celecoxib may be attributed to either stimulation of insulin release or increasing peripheral insulin sensitivity. However, Hsieh et al. ${ }^{27}$ proved that celecoxib reversed fructose-induced increase in insulin level and improved the whole body insulin resistance.

In the present study, administration of glibenclamide $(3 \mathrm{mg} / \mathrm{kg}$, orally) to diabetic rats resulted in significant decrease in the serum glucose level when compared to control diabetic rats with maximum decrease $(31 \%)$ at $4 \mathrm{hrs}$ after its administration. These results were supported by Taj Eldin et al. $^{28}$.

The oral administration of glimepiride (2 $\mathrm{mg} / \mathrm{kg}$ ) to diabetic rats significantly decreased serum glucose level when compared to control diabetic rats. Maximal antidiabetic activity was obtained 3 hrs after administration of glimepiride. These results were in agreement with Niemi et al. ${ }^{29}$.

The combined hypoglycemic activities of celecoxib+ glibenclamide or sitagliptin were found to be approximately equal to the summation of the hypoglycemic activity of each drug alone (i.e. additive). Based on these results, the observed interaction between celecoxib and glibenclamide or sitagliptin may be mainly due to pharmacodynamics interaction which can be attributed to the glucose lowering activity of celecoxib mediated by increasing the peripheral insulin sensitivity.

In celecoxib+ glimepiride combination, the combined hypoglycemic effect was observed to be significantly higher than the summation of the hypoglycemic activity of each drug alone (i.e. synergistic effect) and there was an increase in the hypoglycemic activity duration. This synergistic combined effect could be explained not only by pharmacodynamics interaction, which can be attributed to the hypoglycemic activity of celecoxib, but also may include a 
pharmacokinetic interaction between celecoxib and glimepiride. This has led to conduct a pharmacokinetic study to explore the effect of celecoxib on the pharmacokinetics of glimepiride

Consequently, the effect of celecoxib on glucose uptake by isolated non-diabetic \& diabetic rate pididymal fat pads was studied to investigate its effect on peripheral insulin sensitivity. Peripheral insulin resistance is a key factor in the pathogenesis of T2DM and involves defects in the glucose transport system in both skeletal muscle and adipose tissue. Adipose tissue has a central role in lipid and glucose metabolism and produces a large number of hormones and cytokine ${ }^{30}$. Glucose uptake by isolated rat epididymal fat pads represents a simple and sensitive in-vitro assay where $30-50 \%$ of glucose uptake takes place in adipocytes $^{31}$.

In the present study, in-vitro incubation of isolated epididymal fat pads revealed significant reduction of glucose uptake by incubated fat pads isolated from diabetic rats in comparison with fat pads isolated from nondiabetic rats. These results were confirmed and explained by the study of Camps et al. ${ }^{32}$ who stated that mature fat pads express at least two glucose transporter isoforms, GLUT1 and GLUT4 and the latter was decreased in STZinduced diabetic rats which lead to decrease in media glucose uptake by epididymal fat pads isolated from diabetic rats.

When insulin $(0.1 \mu \mathrm{M})$ was added to the incubated fat pads obtained from non-diabetic or diabetic rats, it significantly stimulated the glucose uptake by 2 - or 2.5-fold, respectively, in comparison with their corresponding groups of non-treated fat pads. This was probably due to the significantly increased GLUT4 recruitment in insulin-treated epididymal fat pads obtained from either non-diabetic or diabetic rats in comparison to non-treated corresponding groups where insulin binds to specific insulin receptor (IR) on the surface of target cells leading to autophosphorylation of intracellular $\beta$-subunit of the IR which in turn result in activation of tyrosine kinase. Activated tyrosine kinase catalyses phosphorylation of insulin receptor substrate protein (IRS-1) which interact with phosphatidyl inositol 3-kinase causing its activation and subsequent recruitment of GLUT4 ${ }^{33}$.

Addition of celecoxib(1.8 or $2.6 \mu \mathrm{M})$ to isolated non-diabetic or diabetic rat epididymal fat pads significantly increased glucose uptake by these tissues when compared to their corresponding non-treated groups. There were significant differences between the effects of either insulin or celecoxib addition on glucose uptake by incubated fat pads isolated from either non-diabetic or diabetic rats. These significant changes induced by either of them were more significantly increased when insulin was added together with celecoxib to the incubated non-diabetic or diabetic rat fat pads as compared to their corresponding groups.

Consequently from this study, it can be deduced that celecoxib was a stimulant of glucose uptake by isolated epididymal fat pads and insulin was not required for celecoxibdependent stimulation of glucose uptake. Also, celecoxib potentiated the insulin-dependent stimulation of glucose uptake and these effects of celecoxibwere more pronounced in diabetic rats than in non-diabetic rats.

Hsieh et $a l^{27}$, showed that celecoxib significantly enhanced insulin-stimulated whole body glucose utilization in normal dietfed or fructose-fed rats, suggesting that celecoxib could also increase insulin sensitivity in normal and insulin resistant rats. Also, the anti-oxidative effects of celecoxib could significantly contribute to its beneficial actions on whole body and muscular insulin resistance because celecoxib reduced a fructose-induced increase in oxidative stress and also suppressed time-dependent increase in oxidative stress in control rats. Some COX-2 inhibitors augment the rate of in-vitro glucose transport indicated by increasing in the GLUT4 mRNA and protein expression in total cell content and the amount of GLUT4 protein in the plasma membrane of L6 myotubes (a rat skeletal myoblast cell line) in an insulin- and AMPactivated protein kinase $\alpha$-independent manner $^{34}$.

The pharmacokinetic study was done to evaluate the effect of celecoxib on the pharmacokinetics of glimepiride. The study of pharmacokinetics of celecoxib was not performed due to absence of significant alteration in the anti-inflammatory activity of celecoxib occurred when celecoxib was 
administrated with glimepiride. The serum concentrations of glimepiride moderately increased by treatment with celecoxib1 $\mathrm{hr}$ after glimepiride administration, and there was significant difference in the peak serum concentration $\left(C_{\max }\right)$ of glimepiride when it was co-administered with celecoxib. The $C_{\max }$ in the serum concentration-time curve of glimepiride+ celecoxib occurred at about $3 \mathrm{hrs,}$ whereas it was seen at $2 \mathrm{hrs}$ post glimepiride administration, indicating that celecoxib may not have affected the rate or the extent of absorption of oral glimepiride considering the significant difference between both the area under the serum concentration-time curve (AUC) and $C_{\max }$ of glimepiride alone and glimepiride + celecoxib-treated group.

Administration of two medications at or around the same time can result in clinically significant drug interactions. Pharmacokinetic drug interactions affect the processes of absorption, distribution, metabolism, and excretion of a drug. The drug-drug interaction at the absorption phase cause difference in time $\left(t_{\max }\right)$ required to reach the same $\mathrm{C}_{\max }{ }^{35}$. Celecoxib did not accelerate the rate of absorption of glimepiride as seen from delayed $t_{\max }$ in this study when co-administrated with celecoxib. Thus the pharmacokinetics interaction between glimepiride and celecoxib may not include the absorption phase.

The elimination rate constant $\left(k_{\mathrm{e}}\right)$ of a drug indicates the proportion of the drug that is removed from the body and half-life $\left(t_{1 / 2}\right)$ is a reciprocal function of $k_{\mathrm{e}}$. In the present study, administration of celecoxib with glimepiride decreased the $k_{\mathrm{e}}$ of the glimepiride especially in linear kinetic and caused an increase in the $t_{1 / 2}$ of glimepiride. The mean $C L / F$ (apparent oral clearance) of glimepiride was decreased while the mean $V_{d} / F$ (apparent volume of distribution) was increased by celecoxib.

Distribution of medications depends on total body water, extracellular fluid, percentage of adipose tissue, and capacity to bind to plasma proteins. Albumin and $\alpha-1$ glycoprotein are the primary circulating plasma proteins to which drugs bind. Some drug-drug interactions occur because of competition for the binding sites on these proteins. One drug knocks the other off the binding site or, alternatively, occupies the site, not allowing the other drug to bind and decreasing its own free fraction.
While the unbound fraction of the other drug is increased and consequently it's pharmacological effect is enhanced ${ }^{35}$.

For an interaction to become clinically significant, the involved drugs must be highly $(>95 \%)$ protein bound or have a very narrow therapeutic index. Celecoxib and glimepiride are highly protein bound $(97.4,99 \%$, respectively) to albumin ${ }^{36 \& 37}$. In this study, the antidiabetic activity of glimepiride was increased by co-administration with celecoxib. On the other hand, the anti-inflammatory activity of celecoxib was not altered when coadministrated with glimepiride indicating that celecoxib did not displace glimepiride from plasma protein.

The liver is the main site of metabolism of glimepiride and the whole drug is cleared from the systemic circulation by the liver. Celecoxibis extensively metabolized, primarily by the CYP2C9 (80\%); while CYP3A4 has a minor role $^{38}$. In this study, the mechanism underlying the interaction between celecoxib and glimepirideis probably competitive inhibition of the CYP2C9-mediated biotransformation of glimepiride by celecoxib. Since celecoxib decreased the clearance of the glimepiride, it may be said that celecoxib altered the metabolism of the glimepiride by the liver. Because celecoxib moderately increased the $t_{1 / 2}$ and $C_{\max }$ of glimepiride, it seems that celecoxib inhibited glimepiride metabolism mainly during the elimination phase and thereby decreased its systemic clearance. The observation that the increase in serum glimepiride concentrations resulted in relatively moderate magnitude of the pharmacokinetic interaction may explain the synergistic increase in the glucose response. Studies of inhibition of glimepiride metabolism with other CYP2C9 substrates were in agreement with the present results ${ }^{39}$.

It can be concluded from this study that celecoxib changed the plasma concentration of glimepiride by competitive inhibition of CYP2C9. Celecoxib expected to not affect CYP3A4 activity because of the absence of pharmacokinetic interaction that one can predict from the additive blood glucose lowering response in case of co-administration of celecoxib and glibenclamide or sitagliptin.

The effect of the three antidiabetic drugs on anti-inflammatory activity of celecoxib was 
studied using carrageenan-induced hind paw edema model in diabetic rats. Intra-plantar injection of carrageenan in diabetic rats showed an inflammatory response that was characterized by an increase in right hind paw weight (indicative of paw edema) when compared with the weight of contralateral (saline-injected) hind paw. These results are in agreement with those obtained $\mathrm{by}^{2}$. In this study, celecoxib(40 mg/kg, orally) administration to diabetic rats $1 \mathrm{hr}$ before carrageenan injection resulted in significant decrease in the paw edema by $44.2 \%$ after $4 \mathrm{hrs}$ of carrageenan injection when compared to control group.

Oral administration of glibenclamide( 3 $\mathrm{mg} / \mathrm{kg}$ ), glimepiride( $2 \mathrm{mg} / \mathrm{kg}$ ) or sitagliptin(3 $\mathrm{mg} / \mathrm{kg}$ ) to diabetic rats $2 \mathrm{hrs}$ before carrageenan injection did not significantly change the paw edema when compared to control group after 4 hrs of carrageenan injection. Oral administration of glibenclamide $(3 \mathrm{mg} / \mathrm{kg}$ ), glimepiride $(2 \mathrm{mg} / \mathrm{kg}$ ) or sitagliptin( $3 \mathrm{mg} / \mathrm{kg}$ ) to diabetic rats $1 \mathrm{hr}$ before administration of celecoxib(40 $\mathrm{mg} / \mathrm{kg}$ ) did not produce any improvement in the paw edema after $4 \mathrm{hrs}$ of carrageenan injection when compared to celecoxib-treated group. The present results showed that glibenclamide, glimepiride and sitagliptin did not possess any antiinflammatory activity in this model. Meanwhile, the three antidiabetic drugs did not-affect neither the pharmacokinetics nor the pharmacodynamics of celecoxib.

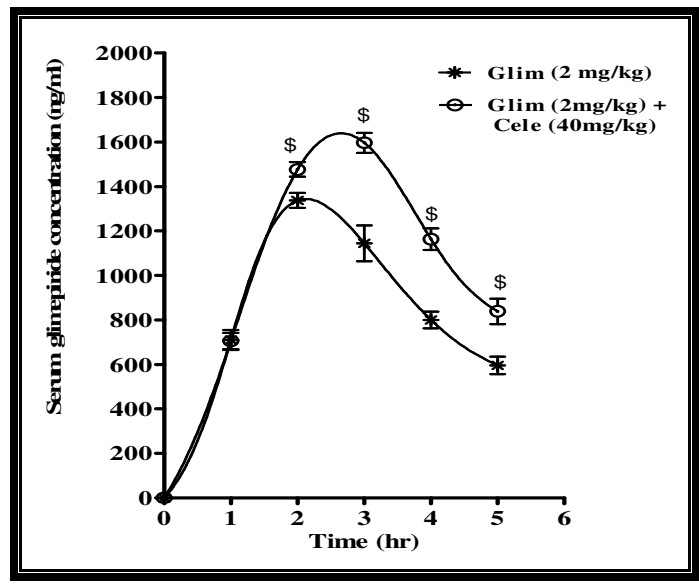

Fig. 3: Effect of single dose of celecoxib (Cele) on serum glimepiride (Glim) concentration in STZ-induced diabetic rats.
Glim (2 mg/kg, orally) was given at time zero, Cele(40 mg/kg, orally) was given after $1 \mathrm{hr}$. Measurement of serum Glimepiridelevels were done before and 1, 2, 3, 4 \& $5 \mathrm{hrs}$ after treatment with Glim.

Values represent the mean \pm SEM of 5 rats.

\$ Significantly different from the corresponding mean value of the Glim-treated group using one way ANOVA followed by Tukey-Kramer multiple comparisons test $(\mathrm{p}<0.05)$.

\section{Conclusion}

Celecoxib potentiated the hypoglycaemic activity of glibenclamide, glimepiride and sitagliptin and the potentiation effect was more pronounced when celecoxib was administrated with glimepiride than with glibenclamide or sitagliptin. Dose adjustment of glibenclamide, glimepiride and sitagliptin must be required when they are co-administrated with celecoxib to avoid the occurrence of sever hypoglycaemia. Glibenclamide, glimepiride and sitagliptin did not have anti-inflammatory activity and did not affect the antiinflammatory activity of celecoxib. Clinical studies should be conducted to evaluate the potentiating effect of celecoxib on the hypoglycaemic activity of glibenclamide, glimepiride and sitagliptin in diabetic patients.

\section{REFERENCES}

1- L. A. Trepanier, "Potential interactions between non-steroidal anti-inflammatory drugs and other drugs", J. Vet. Emerg. Crit. Care, 15, 248-253 (2005).

2- T. D. Penning, J. J. Talley, S. R. Bertenshaw, J. S. Carter, P. W. Collins, S. Docter, M. J. Graneto, L. F. Lee, J. W. Malecha, J. M. Miyashiro, R. S. Rogers, D. J. Rogier, S. S. Yu, G. D. Anderson, E. G. Burton, J. N. Cogburn, S. A. Gregory, C. M. Koboldt,W. E. Perkins, K. Seibert, A. W. Veenhuizen,Y. Y. Zhang and P. C. Isakson, "Synthesis and biological evaluation of the 1,5-diarylpyrazole class of cyclooxygenase-2 inhibitors: Identification of 4-[5-(4-methylphenyl)-3(trifluoromethyl)-1H-pyrazol-1-yl]benze nesulfonamide (SC-58635, celecoxib)", $\boldsymbol{J}$. Med. Chem., 40, 1347-1365 (1997).

3- N. M. Davies, A. J. McLachlan, R. O. Day and K. M. Williams, "Clinical pharmacokinetics and pharmacodynamics 
of celecoxib: A selective cyclo-oxygenase2 inhibitor", Clin. Pharmacokinet., 38, 225-242 (2000).

4- S. A. Grossman, J. Olson, T. Batchelor, D. Peereboom, G. Lesser, S. Desideri, X. Ye, T. Hammour and J. G. Supko, "Effect of phenytoin on celecoxib pharmacokinetics in patients with glioblastoma", Neuro. Oncol., 10, 190-198 (2008).

5- A. Karim, D. S. Tolbert, T. L. Hunt, R. C. Hubbard, K. M. Harper and G. S. Geis, "Celecoxib, a specific COX-2 inhibitor, has no significant effect on methotrexate pharmacokinetics in patients with rheumatoid arthritis", J. Rheumatol., 26, 2539-2543 (1999).

6- U. Werner, D. Werner, T. Rau, M. F. Fromm, B. Hinz and K. Brune, "Celecoxib inhibits metabolism of cytochrome P450 2D6 substrate metoprolol in humans", Clin. Pharmacol. Ther., 74, 130-137 (2003).

7- W. R. Garnett, "Clinical implications of drug interactions with coxibs", Pharmacotherapy, 21, 1223-1232 (2001).

8- R. T. Kubacka, E. J. Antal, R. P. Juhl and I. R. Welshman, "Effects of aspirin and ibuprofen on the pharmacokinetics and pharmacodynamics of glyburide in healthy subjects", Ann. Pharmacother., 30, 20-26 (1996).

9- H. Sone, A. Takahashi and N. Yamada, "Ibuprofen-related hypoglycemia in a patient receiving sulfonylurea", Ann. Intern. Med., 134, 344 (2001).

10- M. Gonzalez-Ortiz, E. Martinez-Abundis, B. R. Balcazar-Munoz and J. A. RoblesCervantes, "Inhibition of cyclooxygenase1 or -2 on insulin sensitivity in healthy subjects", Horm. Metab. Res., 33, 250253 (2001).

11- A. J. Krentz and C. J. Bailey, "Oral antidiabetic agents: Current role in type 2 diabetes mellitus", Drugs, 6, 385-411 (2005).

12- M. M. Goldenberg, "Celecoxib, a selective cyclooxygenase-2 inhibitor for the treatment of rheumatoid arthritis and osteoarthritis", Clin.Ther., 21, 1497-1513 (1999).

13- T. Bobkiewicz-Kozlowska, M. Dworacka, S. Kuczynski, M. Abramczyk, R. Kolanos, W. Wysocka, P. M. Garcia Lopez and H.
Winiarska, "Hypoglycaemic effect of quinolizidine alkaloids-lupanine and 2thionosparteine on non-diabetic and streptozotocin-induced diabetic rats", Eur. J. Pharmacol., 565, 240-244 (2007).

14- Y. J. Hsu,T. H. Lee, C. L. Chang, Y. T. Huang and W. C. Yang, "Antihyperglycemic effects and mechanism of Bidenspilosa water extract", $\boldsymbol{J}$. Ethnopharmacol., 122, 379-383 (2009).

15- A. Tahara, A. Matsuyama-Yokono, R. Nakano, Y. Someya and M. Shibasaki, "Hypoglycaemic effects of antidiabetic drugs in streptozotocin-nicotinamideinduced mildly diabetic and streptozotocin-induced severely diabetic rats", Basic Clin. Pharmacol. Toxicol., 103, 560-568 (2008).

16- C. A. Winter, E. A. Risley and G. W. Nuss, "Carrageenin-induced edema in hind paw of the rat as an assay for antiiflammatory drugs", Proc. Soc. Exp. Biol. Med., 111, 544-547 (1962).

17- P. Trinder, "Determination of blood glucose using 4-amino phenazone as oxygen acceptor", J. Clin. Pathol., 22, 246 (1969).

18- M. Rodbell, "Metabolism of isolated fat cells. i. effects of hormones on glucose metabolism and lipolysis", J. Biol. Chem., 239, 375-380 (1964).

19- M. Vaughan, "The production and release of glycerol by adipose tissue incubated invitro", ibid., 237, 3354-3358 (1962).

20- C. B. Graves, R. D. Gale, J. P. Laurino and J. M. McDonald, "The insulin receptor and calmodulin. Calmodulin enhances insulin-mediated receptor kinase activity and insulin stimulates phosphorylation of calmodulin", ibid., 261, 10429-10438 (1986).

21- L. Rabbaa-Khabbaz, R. biDaoud, D. Karam-Sarkis, C. Atallah and A. A. Zoghbi, "Simple and sensitive method for determination of glimepiride in human serum by HPLC", J. Liq. Chromatogr. Relat. Technol., 28, 3255-3263 (2005).

22- L. Ladriere, F. Malaisse-Lagae, J. Fuhlendorff and W. J. Malaisse, "Repaglinide, glibenclamide and glimepiride administrationto normal and 
hereditarily diabetic rats", Eur. J. Pharmacol., 335, 227-234 (1997).

23- T. Cicchini, M. Belli, P. Antonioni, P. Cicchini and L. Maddalena, "Behaviour of glycemia in rats inoculated with prostaglandin", Ann. Sclavo., 22, 157-164 (1980).

24- D. Giugliano, R. Torella, A. J. Scheen, P. J. Lefebvre and F. D'Onofrio, "Prostaglandins, insulin secretion and diabetes mellitus", Diabete Metab., 14, 721-727 (1988).

25- M. Gonzalez-Ortiz, S. Pascoe-Gonzalez, A. Esperanzamartinez, A. M. Kam-Ramos and E. Hernandez-Salazar, "Effect of celecoxib, a cyclooxygenase-2-specific inhibitor, on insulin sensitivity, C-reactive protein, homocysteine, and metabolic profile in overweight or obese subjects", Metab. Syndr. Relat. Disord., 3, 95101(2005).

26- S. D. Long and P. H. Pekala, "Regulation of GLUT4 gene expression by arachidonic acid. Evidence for multiple pathways, one of which requires oxidation to prostaglandin E2", J. Biol. Chem., 271, 1138-1144 (1996).

27- P. S. Hsieh, H. C. Tsai, C. H. Kuo, J. Y. Chan, J. F. Shyu, W. T. Cheng and T. T. Liu, "Selective COX2 inhibition improves whole body and muscular insulin resistance in fructose-fed rats", Eur. J. Clin. Invest., 38, 812-819 (2008).

28- I. M. Taj Eldin, E. M. Ahmed and H. M. Abd Elwahab, "Preliminary study of the clinical hypoglycemic effects of Allium cepa (red onion) in Type 1 and Type 2 biabetic patients", Environ. Health Insights., 4, 71-77 (2010).

29- M. Niemi, K. T. Kivisto, J. T. Backman and P .J. Neuvonen, "Effect of rifampicin on the pharmacokineticsand pharmacodynamics of glimepiride", Br. J. Clin. Pharmacol., 50, 591-595 (2000).

30- L. Maianu, S. R. Keller and W. T. Garvey, "Adipocytes exhibit abnormal subcellular distribution and translocation of vesicles containing glucose transporter 4 and insulin-regulated aminopeptidase in type 2 diabetes mellitus: Implications regarding defects in vesicle trafficking", J. Clin. Endocrinol. Metab., 86, 5450-5456 (2001).
31- M. Haag, A. C. Malipa, D. H. van Papendorp, P. I. Stivaktas, S. Alummoottil and A. M. Koorts, "Short-term fatty acid effects on adipocyte glucose uptake: Mmechanistic insights", Prostaglandins Leukot. Essent. Fatty Acids, 80, 247-253 (2009).

32- M. Camps, A. Castello, P. Munoz, M. Monfar, X. Testar, M. Palacin and A. Zorzano, "Effect of diabetes and fasting on GLUT-4 (muscle/fat) glucosetransporter expression in insulin-sensitive tissues. Heterogeneous response in heart, red and white muscle", Biochem. J., 282, 765-772 (1992).

33- L. Chang, S. H. Chiang and A. R. Saltiel, "Insulin signaling and the regulation of glucose transport", Mol. Med., 10, 65-71 (2004).

34- E. Alpert, A. Gruzman, B. Lardi-Studler, G. Cohen, R. Reich and S. Sasson, "Cyclooxygenase-2 (PTGS2) inhibitors augment the rate of hexose transport in L6 myotubes in an insulin- and AMPK alphaindependent manner", Diabetologia, 49, 562-570 (2006).

35- S. F. Manzi and M. Shannon, "Drug Interactions: A Review", Clin. Ped. Emerg. Med., 6, 93-102 (2005).

36- M. Badian, A. Korn, K. H. Lehr, V. Malerczyk and W. Waldhausl, "Absolute bioavailability of glimepiride (Amaryl) after oral administration", Drug Metab. Drug Interact., 11, 331-339 (1994).

37- K. Brune and B. Hinz, "Selective cyclooxygenase-2 inhibitors: Similarities and differences", Scand. J. Rheumatol., 33, 1-6 (2004).

38- C. Tang, M. Shou, Q. Mei, T. H. Rushmore and A. D. Rodrigues, "Major role of human liver microsomal cytochrome P450 2C9 (CYP2C9) in the oxidative metabolism of celecoxib, a novel cyclooxygenase-II inhibitor", $\boldsymbol{J}$. Pharmacol. Exp. Ther., 293, 453-459 (2000).

39- V. J. Galani and M. Vyas, "In-vivo and Invitro drug interactions study of glimepride with atorvastatin and rosuvastatin", $\boldsymbol{J}$. Young Pharm., 2, 196-200 (2010). 


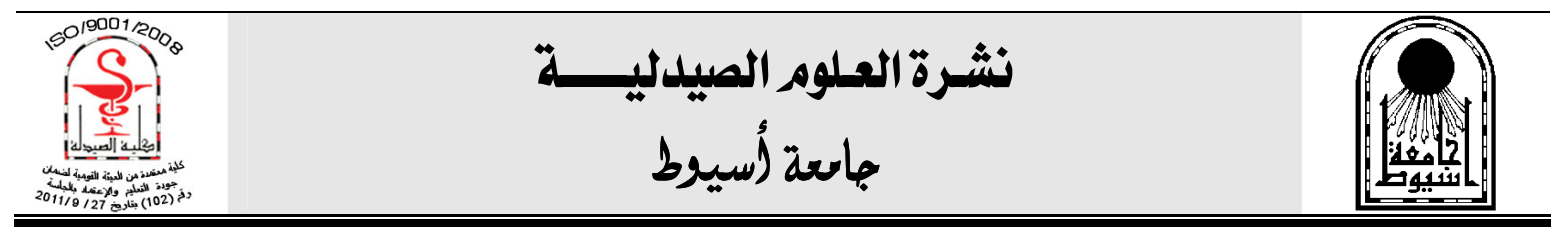

التفاعلاث التاخلية للسليكوكسيب مع الأدوية الفموية المختلفة المضادة للسكري في

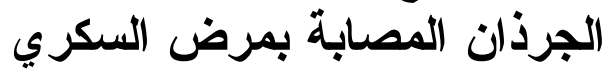

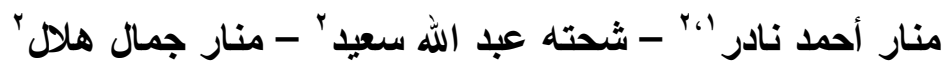

\section{' قسم الفارماكولوجي والسموم ، كلية الصيدلة ، جامعة طيبه ، المدينه المنورة ، المملكة العربية السعودية

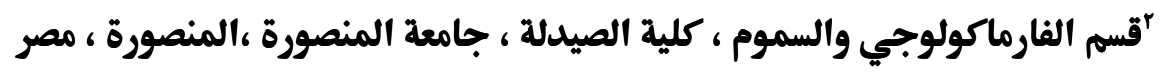

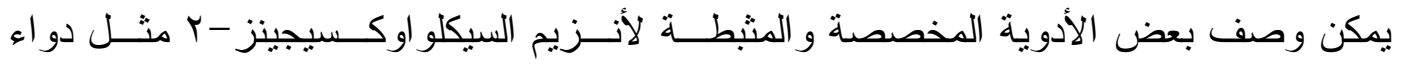

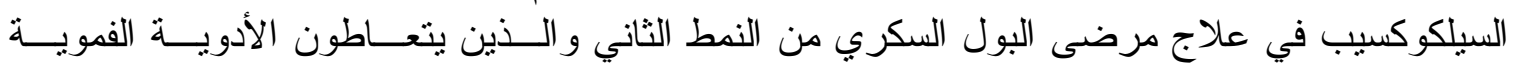

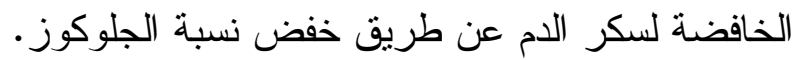

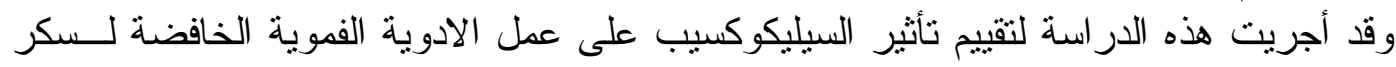

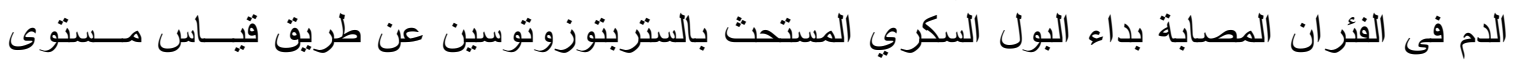

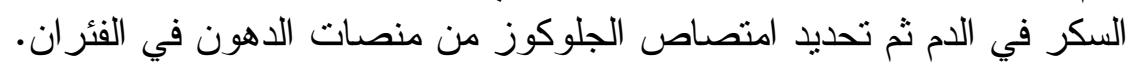

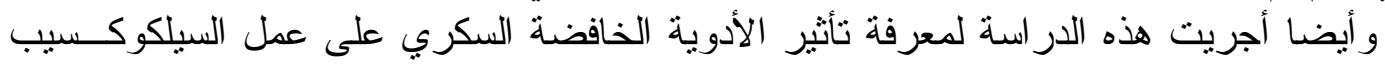

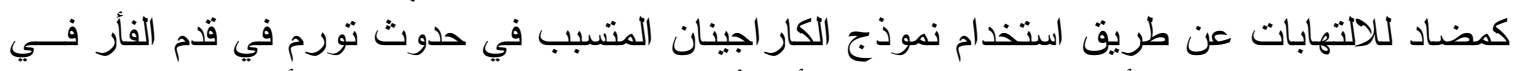

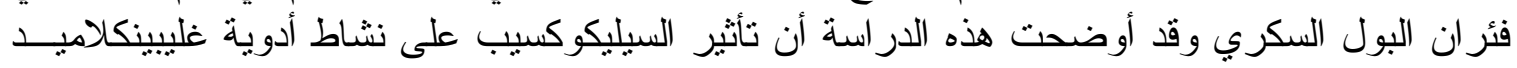

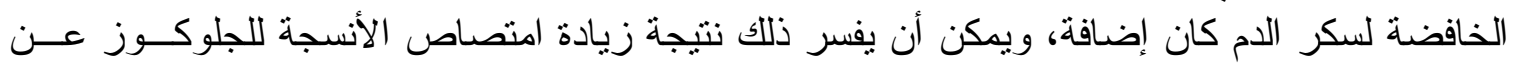

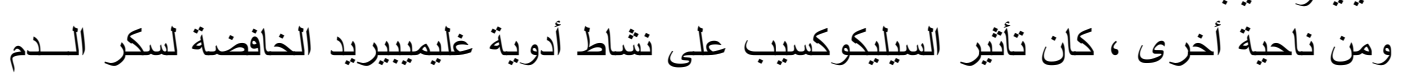

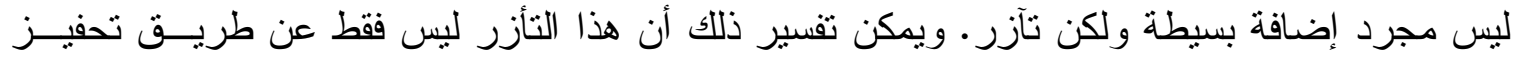

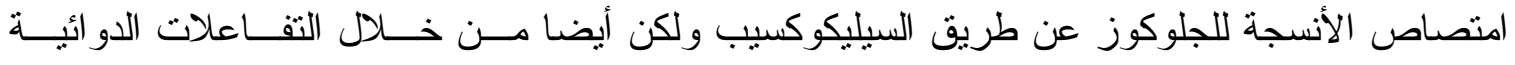
الحركية عن طريق زيادة نسبة غليميبيريد في الدم. لانئ 